Pacific Journal of Mathematics

NONPOSITIVELY CURVED HOMOGENEOUS SPACES OF 


\title{
NONPOSITIVELY CURVED HOMOGENEOUS SPACES OF DIMENSION FIVE
}

\author{
María J. Druetta
}

\begin{abstract}
In this paper we classify, in terms of the rank, the simply connected homogeneous spaces of nonpositive curvature and dimension five. In particular, an affirmative answer is given to the conjecture "An irreducible homogeneous space of nonpositive curvature and rank $k \geq 2$ is a symmetric space of rank $k$ ".

We exhibit examples in dimension five of rank one homogeneous spaces of nonpositive curvature having totally geodesic two-flats isometrically imbedded. Moreover, these examples show that the rank in a Lie group is not invariant under the change of left invariant metrics of nonpositive curvature
\end{abstract}

Introduction. In this paper we study, in terms of the rank, the simply connected Lie groups $G$ of dimension five with left invariant metrics of nonpositive curvature $(K \leq 0)$. The results obtained are then used to get a classification of the simply connected homogeneous spaces of nonpositive curvature of rank two and dimension five. We exhibit on $G$, the Lie group of $3 \times 3$ upper triangular real matrices of determinant one, many different left invariant metrics of $K \leq 0$ and rank one. We remark that $G$ also has a unique, up to a positive constant factor, left invariant metric of $K \leq 0$ and rank two which turns it into a symmetric space. Thus we obtain examples of rank one homogeneous spaces of nonpositive curvature having two-flats isometrically embedded. Moreover, we show that a Lie group (of dimension five) may admit different left invariant metrics of nonpositive curvature of different ranks.

In $\S 1$ we classify the simply connected five-dimensional homogeneous spaces $H$ of nonpositive curvature with no flat de Rham factor and rank two. We show that, either $H=H^{2} \times T^{3}$ where $H^{2}$ is a twodimensional space of constant negative curvature and $T^{3}$ is a rank one homogeneous space of $K \leq 0$, or $H=\mathrm{SL}(3, \mathbf{R}) / \mathrm{SO}(3)$ the irreducible symmetric space of noncompact type and rank two, provided that we multiply the metric by a suitable positive constant.

Section 2 is an auxiliary section needed to complete the classification given in $\S 1$. Here, we study a particular example in dimension five that 
corresponds to studying all the left invariant metrics of $K \leq 0$ on the group $G$ of $3 \times 3$-upper triangular real matrices of determinant one.

In $\S 3$ we exhibit many different metrics turning $G$ into rank one homogeneous spaces having 2-flats isometrically imbedded. Furthermore, a comparison result between the symmetric metric on $G$ and non-symmetric ones is obtained.

Preliminaries. Let $H$ be a complete simply connected Riemannian manifold of nonpositive curvature $(K \leq 0)$. If $\gamma$ is a unit speed geodesic in $H, \operatorname{rank}(\gamma)$ is defined to be the dimension of the vector space of all parallel Jacobi fields along $\gamma$. The minimum of $\operatorname{rank}(\gamma)$ over all geodesics $\gamma$ of $H$ is called rank of $H$ and denoted by $\operatorname{rank}(H)$. This definition was introduced in [3] and coincides with the usual one if $H$ is a symmetric space.

Assume that $H$ is a homogeneous space. Then $\operatorname{rank}(H)$ is the minimum of $\operatorname{rank}(\gamma)$ over all geodesics $\gamma$ of $H$ such that $\gamma(0)=p$ for some $p$ in $H$. In this case, $H$ admits a simply transitive and solvable group of isometries (see [1]) and hence, $H$ can be represented as a solvable Lie group $G$ with a left invariant metric of nonpositive curvature.

Given a Lie group $G$ with Lie algebra $\mathfrak{g}$ and left invariant metric $\langle$,$\rangle , we recall that if X, Y, Z \in \mathfrak{g}$ then the Riemannian connection $\nabla$ is given by

$$
2\left\langle\nabla_{X} Y, Z\right\rangle=\langle[X, Y], Z\rangle-\langle[Y, Z]\rangle+\langle[Z, X], Y\rangle .
$$

If $R(X, Y)=\left[\nabla_{X}, \nabla_{Y}\right]-\nabla_{[X, Y]}$ is the curvature tensor associated to $\nabla$, the sectional curvature $K$ is given by

$$
\begin{aligned}
& |X \wedge Y|^{2} K(X, Y)=\langle R(X, Y) Y, X\rangle \\
& \quad=\frac{1}{4}|U(X, Y)|^{2}-\frac{1}{4}\langle U(X, X), U(Y, Y)\rangle-\frac{3}{4}|[X, Y]|^{2} \\
& \quad-\frac{1}{2}\langle[[X, Y], Y], X\rangle-\frac{1}{2}\langle[[Y, X], X], Y\rangle
\end{aligned}
$$

where $U(X, Y)=\left(\operatorname{ad}_{X}\right)^{*} Y+\left(\operatorname{ad}_{Y}\right)^{*} X$, and $\left(\operatorname{ad}_{X}\right)^{*}$ denotes the adjoint of $\operatorname{ad}_{X}$.

Let $G$ be a solvable simply connected Lie group with a left invariant metric of nonpositive curvature. If $\mathfrak{a}$ is the orthogonal complement of $[\mathfrak{g}, \mathfrak{g}]$ in $\mathfrak{g}$ with respect to the metric, it follows from [1, Theorem 5.2] that it is an abelian subalgebra of $\mathfrak{g}$ which is also totally geodesic $\left(\nabla_{X} Y \in \mathfrak{a}\right.$ for all $\left.X, Y \in \mathfrak{a}\right)$. Moreover, $A=\exp \mathfrak{a}$, the connected Lie subgroup of $G$ with Lie algebra $\mathfrak{a}$, is a $\operatorname{dim} \mathfrak{a}$-flat in $G$.

In general, a $k$-flat in $H$ is defined to be the image of a totally geodesic isometric imbedding of $R^{k}$ into $H$. 
1. Homogeneous spaces of $K \leq 0$ and dimension five. In this section we characterize, in terms of rank, the simply connected homogeneous spaces of nonpositive curvature $(K \leq 0)$ and dimension five.

Let $G$ be a solvable and simply connected Lie group with a left invariant metric of nonpositive curvature. If $\mathfrak{g}$ is the Lie algebra of $G$, then $\mathfrak{g}=[\mathfrak{g}, \mathfrak{g}] \oplus \mathfrak{a}$ where $\mathfrak{a}$, the orthogonal complement of $[\mathfrak{g}, \mathfrak{g}]$ with respect to the metric, is an abelian subalgebra of $\mathfrak{g}$.

If $\mathfrak{g}^{\prime \mathbf{c}}$ is the complexification of $\mathfrak{g}^{\prime}=[\mathfrak{g}, \mathfrak{g}]$ then we have a direct sum decomposition $\mathfrak{g}^{\prime \mathbf{c}}=\sum_{\lambda} \mathfrak{g}_{\lambda}^{\prime \mathbf{c}}$, where

$$
\mathfrak{g}_{\lambda}^{\prime \mathbf{c}}=\left\{U \in \mathfrak{g}^{\prime \mathbf{c}}:\left(\operatorname{ad}_{H}-\lambda(H) I\right)^{k} U=0\right.
$$

for some $k \geq 1$ and for all $H \in \mathfrak{a}\}$

is the associated root space for the root $\lambda \in\left(\mathfrak{a}^{*}\right)^{\mathbf{c}}$ under the abelian action of $\mathfrak{a}$ on $\mathfrak{g}^{\prime}$. If $\lambda=\alpha \pm i \beta$ is a root of $\mathfrak{a}$ in $\mathfrak{g}^{\prime}$ (that is, $\mathfrak{g}_{\lambda}^{\prime \mathbf{c}} \neq 0$ ), the generalized root space is defined by $\mathfrak{g}_{\alpha, \beta}^{\prime}=\mathfrak{g}_{\alpha,-\beta}^{\prime}=\mathfrak{g}^{\prime} \cap\left(\mathfrak{g}_{\lambda}^{\prime \mathbf{c}} \oplus \mathfrak{g}_{\lambda}^{\prime \mathbf{c}}\right)$ and $\mathfrak{g}^{\prime}$ is the direct sum of the $\operatorname{ad}_{\mathfrak{a}}$-invariant subspaces $\mathfrak{g}_{\alpha, \beta}^{\prime}$.

We assume that $G$ has no de Rham flat factor. Then, it follows from $\left[2\right.$, Theorem 4.6] that the above condition is equivalent to $\mathfrak{g}_{0}^{\prime}=\sum_{\mathfrak{g}_{0, \beta}^{\prime}}$ and $\mathfrak{a}_{0}=\{H \in \mathfrak{a}: \alpha(H)=0$ for all roots $\alpha+i \beta\}$ are zero.

The following formulas about sectional curvatures will be used frequently; we include the proofs for the sake of completeness. In the sequel, if $H \in \mathfrak{a}$ we will denote by $D_{H}$ and $S_{H}$ the symmetric and skew-symmetric part of $\operatorname{ad}_{H}$ respectively with respect to the metric $\langle$,$\rangle .$

Lemma 1.1. Assume $\mathfrak{g}^{\prime}$ abelian.

(i) Let $\left\{H_{i}\right\}_{i=1}^{k}$ be an orthonormal basis for a and set $D_{i}=D_{H_{i}}$, $i=1, \ldots, k$. Then,

$$
\langle R(X, Y) Y, X\rangle=\sum_{i=1}^{k}\left(\left\langle D_{i} X, Y\right\rangle^{2}-\left\langle D_{i} X, X\right\rangle\left\langle D_{i} Y, Y\right\rangle\right)
$$

for all $X, Y \in \mathfrak{g}^{\prime}$.

(ii) $\langle R(X, Y+H)(Y+H), X\rangle=\langle R(X, Y) Y, X\rangle+\langle R(X, H) H, X\rangle$ for all $X, Y \in \mathfrak{g}^{\prime}$ and $H \in \mathfrak{a}$.

In general, we have $\langle R(X, H) H, X\rangle=\left|S_{H} X\right|^{2}-|[H, X]|^{2}$ Lemma 3.4]).

Proof. Let $X, Y \in \mathfrak{g}^{\prime}$ and $H \in \mathfrak{a}$. 
(i) We note that since $\mathfrak{g}^{\prime}$ is abelian, $U(X, Y) \in \mathfrak{a}$ and $\langle U(X, Y)$, $H\rangle=-2\left\langle D_{H} X, Y\right\rangle$. Hence, $U(X, Y)=-2 \sum_{i=1}^{k}\left\langle D_{i} X, Y\right\rangle H_{i}$; the assertion follows from the curvature formula.

(ii) Since $\nabla_{H} X \in \mathfrak{g}^{\prime}$ and $\nabla_{X} Y \in \mathfrak{a}$ we have $R(X, Y) H \in \mathfrak{a}$; from this (ii) follows easily.

REMARK 1.2. If there exists an orthonormal basis $\left\{H_{i}\right\}_{i=1}^{k}$ of $\mathfrak{a}$ such that $D_{i}(i=1, \ldots, k)$ are all positive semidefinite, we have $K(X, Y) \leq 0$ for all $X, Y$ independent in $\mathfrak{g}^{\prime}$. Moreover, we get $K(X, Y)<0$ if for some $j=1, \ldots, k, D_{i}$ is positive definite.

THEOREM 1.3. Let $H$ be a simply connected homogeneous space of nonpositive curvature and $\operatorname{dim} H=5$. If $H$ has no de Rham flat factor then, either $\operatorname{rank}(H)=1$ or $\operatorname{rank}(H)=2$ and it is one of the following spaces

(i) $H=H^{2} \times T^{3}$, where $H^{2}$ is a two-dimensional space of constant negative curvature and $T^{3}$ is a rank one homogeneous space of nonpositive curvature.

(ii) $H=\mathrm{SL}(3, \mathbf{R}) / \mathrm{SO}(3)$, the irreducible symmetric space of noncompact type and rank two, up to multiplying the metric by a positive constant.

We recall that in a three dimensional homogeneous space of nonpositive curvature, rank one and the visibility axiom are equivalent. These spaces were completely characterized in [6] (see Corollary 2.5 and Remark 4.3).

Proof. Let $G$ be a solvable Lie group that acts simply and transitively on $H$. Then, we may assume that $H=G$ is a solvable and simply connected Lie group of dimension five with a left invariant metric of $K \leq 0$ with no flat de Rham factor.

Let $\mathfrak{g}=\mathfrak{g}^{\prime} \oplus \mathfrak{a}, \mathfrak{a}$ the orthogonal complement of $\mathfrak{g}^{\prime}$ with respect to the metric $\langle$,$\rangle . We only need to consider the case \operatorname{dim} \mathfrak{a}=$ 2. In fact, in the case $\operatorname{dim} \mathfrak{a}=1$ it follows from [7, Theorem 1.5] that $G$ has rank one. If $\operatorname{dim} \mathfrak{a}=3$, there exist at most two roots of $\mathfrak{a}$ in $\mathfrak{g}^{\prime}\left(\operatorname{dim} \mathfrak{g}^{\prime}=2\right)$ and consequently we may choose $H \in \overline{\mathfrak{a}}$. satisfying $\alpha(H)=0$ for all $\alpha$ with $\alpha+i \beta$ root; this implies that $G$ has de Rham flat factor (see the remark at the beginning of this section). If $\operatorname{dim} \mathfrak{a}=4, \mathfrak{g}$ is the example given in [6, Example 3.4] and $G$ is isometric to $R^{3} \times H^{2}$. 
Henceforth we assume that $\operatorname{dim} \mathfrak{a}=2$. Note that counting according to multiplicities, there are three roots of $\mathfrak{a}$ on $\mathfrak{g}^{\prime}$. Their real parts span the dual space $\mathfrak{a}^{*}$ (otherwise $\mathfrak{a}_{0}$ would be nonzero). Thus there are two cases: either

(1) two real parts are proportional and the third is independent of them, or

(2) the three real parts (necessarily roots) are pairwise independent. We first show the following lemma.

LEMMA. If $\mathfrak{g}^{\prime}$ is not abelian, then $\mathfrak{a}$ has three real roots $\lambda_{1}, \lambda_{2}$ and $\lambda_{3}$ on $\mathfrak{g}^{\prime}$ such that $\lambda_{1}$ and $\lambda_{2}$ are independent and $\lambda_{3}=\lambda_{1}+\lambda_{2}$. Moreover the center $\mathfrak{z}$ of $\mathfrak{g}^{\prime}$ is the root space of $\lambda_{3}$.

Proof. Note that $\mathfrak{z} \neq 0$ because $\mathfrak{g}$ is solvable and hence $\mathfrak{g}^{\prime}$ is nilpotent. Since $\mathfrak{z}$ is one-dimensional and $\operatorname{ad}_{\mathfrak{a}}$-invariant we have $\mathfrak{z}=\mathfrak{g}_{\lambda}^{\prime}$, the root space associated to a nonzero real root $\lambda\left(\mathfrak{g}_{0}^{\prime}=0\right)$. We observe that there is no complex root $\gamma=\alpha+i \beta, \alpha \neq 0$; if this is the case, $\mathfrak{g}^{\prime \mathbf{c}}=\mathfrak{g}_{\lambda}^{\prime \mathbf{c}} \oplus \mathfrak{g}_{\bar{\gamma}}^{\prime \mathbf{c}} \oplus \mathfrak{g}_{\lambda}^{\prime \mathbf{c}}$ with $0 \neq\left[\mathfrak{g}_{\gamma}^{\prime \mathbf{c}}, \mathfrak{g}_{\bar{\gamma}}^{\prime \mathbf{c}}\right] \subset \mathfrak{g}_{\gamma+\bar{\gamma}}^{\prime \mathbf{c}}=\mathfrak{g}_{2 \alpha}^{\prime \mathbf{c}}$. Thus $\lambda=2 \alpha$, implying that $G$ has de Rham flat factor. Hence, since $\mathfrak{g}^{\prime}$ is not abelian we have real roots $\lambda_{1}, \lambda_{2}$ and $\lambda_{1}+\lambda_{2}\left(0 \neq\left[\mathfrak{g}_{\lambda_{1}}^{\prime}, \mathfrak{g}_{\lambda_{2}}^{\prime}\right] \subset \mathfrak{g}_{\lambda_{1}+\lambda_{2}}^{\prime}\right)$ where $\lambda_{1}$ and $\lambda_{2}$ are independent.

Case 1. The lemma shows that $\mathfrak{g}^{\prime}$ is abelian. It follows from the direct sum decomposition of $\mathfrak{g}^{\prime}$ in generalized root spaces that there is an $\operatorname{ad}_{\mathfrak{a}}$ invariant orthogonal direct sum decomposition $\mathfrak{g}^{\prime}=\mathfrak{g}_{1}^{\prime} \oplus \mathfrak{g}_{2}^{\prime}$ (see $[1, \S 5.3])$ in which

(i) $\mathfrak{g}_{i}^{\prime}$ has dimension $i(i=1,2)$.

(ii) There is a basis $\{\gamma, \alpha\}$ of $\mathfrak{a}^{*}$ such that $\gamma$ is the (necessarily real) root of $\mathfrak{a}$ on $\mathfrak{g}_{1}^{\prime}$ and the real part of every root of $\mathfrak{a}$ on $\mathfrak{g}_{2}^{\prime}$ is proportional to $\alpha$.

We define $H_{1}, H_{2} \in \mathfrak{a}$ by $\gamma(H)=\left\langle H, H_{1}\right\rangle$ and $\alpha(H)=\left\langle H, H_{2}\right\rangle$ for all $H \in \mathfrak{a}$. It follows from Lemma 5.4 (iv) of [1] that $\left\langle H_{1}, H_{2}\right\rangle \geq$ 0 . Thus, there are two cases to consider: either

$$
\left\langle H_{1}, H_{2}\right\rangle=0
$$

or

$$
\left\langle H_{1}, H_{2}\right\rangle>0 \text {. }
$$

Case 1.1. In this case it turns out that $G$ is isometric to a Riemannian product. Let $\mathfrak{t}=\mathfrak{g}_{2}^{\prime} \oplus \mathbf{R} H_{2}$ and $\mathfrak{h}=\mathfrak{g}_{1}^{\prime} \oplus \mathbf{R} H_{1}$. Then $\mathfrak{t}$ is an 
ideal of $\mathfrak{g}, \mathfrak{h}$ is a subalgebra, and $\mathfrak{g}$ is the orthogonal direct sum of $\mathfrak{t}$ and $\mathfrak{g}$. Note that $\operatorname{ad}_{H_{1} \mid \mathfrak{g}_{2}^{\prime}}$ is almost normal and has purely imaginary eigenvalues because $\alpha\left(H_{1}\right)=\left\langle H_{1}, H_{2}\right\rangle=0$. It follows from Lemma 4.4 of [1] that $\operatorname{ad}_{H_{1} \mid \mathfrak{g}_{2}^{\prime}}$ is skew symmetric. Since $\mathfrak{g}^{\prime}$ and $\mathfrak{a}$ are abelian, it now follows that $\operatorname{ad}_{X \mid \mathfrak{t}}$ is skew symmetric for every $X \in \mathfrak{h}$. Hence, $G$ is isometric to the Riemannian product $T^{3} \times H^{2}$ where $T^{3}$ and $H^{2}$ are the connected Lie subgroups of $G$ with Lie algebras $\mathfrak{t}$ and $\mathfrak{h}$ respectively, and left invariant metric induced by the one of $\mathfrak{g}$ (see [6, Lemma 4.1]). Moreover, $H^{2}$ has sectional curvature $K=K\left(e_{1}, H_{1}\right)=-\left|H_{1}\right|^{2}\left(e_{1}\right.$ is a unit vector in $\left.\mathfrak{g}_{1}^{\prime}\right)$ and $T^{3}$ is a rank one homogeneous space of $K \leq 0$ since it has no flat de Rham factor (see [7, Theorem 1.5]).

Case 1.2. In this case it turns out that $G$ has rank one. We will prove this in the two following steps:

(1) $\langle R(X, Y) Y, X\rangle<0$ whenever $X, Y \in \mathfrak{g}^{\prime}$ are independent.

(2) There is $X \in \mathfrak{g}^{\prime}$ with $\langle R(X, H) H, X\rangle<0$ for all nonzero $H \in \mathfrak{a}$.

Hence, applying Lemma 1.1-(ii) we get $K(X, Y+H)<0$ for all $Y$ independent of $X$ in $\mathfrak{g}^{\prime}$ and all $H \in \mathfrak{a}$; consequently the geodesic $\gamma$ in $G$ satisfying $\gamma(0)=e, \gamma^{\prime}(0)=X$ has rank one and therefore $\operatorname{rank}(G)=1$.

Step 1. This will be done by showing that $D_{H_{2}}$ is positive definite and the unit vector $H_{0} \in \mathfrak{a}$ with $\left\langle H_{0}, H_{2}\right\rangle=0$ and $\left.\left\langle H_{0}, H_{1}\right\rangle\right\rangle$ 0 gives $D_{H_{0}}$ positive semidefinite. Then by applying Remark 1.2, assertion (1) follows.

Note that the choice of $H_{0}$ means that $\operatorname{ad}_{H_{0}}$ has a positive eigenvalue on the one-dimensional space $\mathfrak{g}_{1}^{\prime}$ and has purely imaginary eigenvalues on $\mathfrak{g}_{2}^{\prime}\left(\gamma\left(H_{0}\right)=\left\langle H_{0}, H_{1}\right\rangle>0\right.$ and $\alpha\left(H_{0}\right)=\left\langle H_{0}, H_{1}\right\rangle=$ $0)$. By the argument explained above in Case 1.1, one sees that $\operatorname{ad}_{H_{0}}$ is skew symmetric on $\mathfrak{g}_{2}^{\prime}$. Thus, $D_{H_{0}}$ vanishes on $\mathfrak{g}_{2}^{\prime}$ and hence it is positive semidefinite on $\mathfrak{g}^{\prime}$.

Since $\left\langle H_{1}, H_{2}\right\rangle>0$, it follows that $D_{H_{2}}$ is positive definite on $\mathfrak{g}_{1}^{\prime}$. It remains to show that $D_{H_{2}}$ is positive definite on $\mathfrak{g}_{2}^{\prime}$. We observe first that if $c \alpha$ is the real part of a root of $\mathfrak{a}$ on $\mathfrak{g}^{\prime}$ it follows from Lemma 5.4 (iv) of [1] that $c>0$ ( $\mathfrak{g}^{\prime}$ is abelian). Hence, both eigenvalues of $\operatorname{ad}_{H_{2} \mid \mathfrak{g}_{2}^{\prime}}$ have positive real part and since $\operatorname{Tr}\left(D_{H_{2} \mid \mathfrak{g}_{2}^{\prime}}\right)=\operatorname{Tr}\left(\operatorname{ad}_{H_{2} \mid \mathfrak{g}_{2}^{\prime}}\right)>0$, we have that $D_{H_{2} \mid g_{2}^{\prime}}$ cannot be negative definite. Thus, it suffices to prove that $D_{H_{2} \mid g_{2}^{\prime}}$ is definite. If this is not the case, then there is 
$X \in \mathfrak{g}_{2}^{\prime}$ with $D_{H_{2}} X=0$. Since $D_{H_{0}}$ vanishes on $\mathfrak{g}_{2}^{\prime}$, it follows that $D_{H} X=0$ for all $H \in \mathfrak{a}$, which is impossible because the only oneparameter subgroups which are geodesics are $\exp t H, H \in \mathfrak{a}($ see $[8$, Theorem 3.6]).

Step 2. Since $D_{H_{2}}$ is positive definite, we can choose a nonzero vector $e_{2} \in \mathfrak{g}_{2}^{\prime}$ such that $D_{H_{2}} e_{2}$ is a nonzero multiple of $e_{2}\left(D_{H_{2} \mid g_{2}^{\prime}}\right.$ is symmetric). Let $e_{1}$ be a nonzero vector in $g_{1}^{\prime}$ and let $X=e_{1}+e_{2}$. For any $H \in \mathfrak{a}, D_{H} e_{1}$ and $D_{H} e_{2}$ are orthogonal, and $D_{H} e_{1}=0$, $D_{H} e_{2}=0$ if and only if $H$ is orthogonal to $H_{1}$, and $H$ is a multiple of $H_{0}$ respectively ( $H_{0}$ is the same as in Step 1$)$. Since $\left\langle H_{0}, H_{2}\right\rangle=0$ and $H_{1}, H_{2}$ are independent, it follows that $D_{H} X \neq 0$ for all nonzero $H \in \mathfrak{a}$.

Now, we observe that $\left\langle D_{H} X, S_{H} X\right\rangle=0$ for all $H \in \mathfrak{a}\left(S_{H} e_{1}=\right.$ $0, D_{H_{0} \mid g_{2}^{\prime}}=0, D_{H_{2}} e_{2}$ is a multiple of $\left.e_{2}\right)$.

Hence $K(X, H)=\left|S_{H} X\right|^{2}-|[H, X]|^{2}=-\left|D_{H} X\right|^{2}<0$ for all nonzero $H \in \mathfrak{a}$.

Case 2. We will show that either $G$ has rank one or $G$ is an irreducible symmetric space of rank two.

Case 2.1. $\mathfrak{g}^{\prime}$ abelian with three pairwise independent real roots $\lambda_{1}, \lambda_{2}$ and $\lambda_{3}$.

We prove next that $G$ has rank one. By permuting $\lambda_{1}, \lambda_{2}$ and $\lambda_{3}$, one can assume that $\lambda_{3}=a \lambda_{1}+b \lambda_{2}$ with both $a$ and $b$ positive. In fact, we define $H_{i} \in \mathfrak{a}$ by $\lambda_{i}(H)=\left\langle H, H_{i}\right\rangle(i=1,2,3)$ for all $H \in \mathfrak{a}$. Then the $H_{i}$ 's are three nonzero vectors in the twodimensional space $\mathfrak{a}$ and since $\left\langle H_{i}, H_{j}\right\rangle \geq 0$ (see [1, Lemma 5.4(iv)]) the angle between any two of them is at most $\pi / 2$. We assign the indices so that $H_{1}$ and $H_{2}$ are the two outer vectors and $H_{3}$ lies in between.

Since $\mathfrak{g}_{\lambda_{i}}^{\prime}(i=1,2,3)$, the root space associated to $\lambda_{i}$, is onedimensional and the roots $\lambda_{i}$ are pairwise independent, we have an orthonormal basis $\left\{e_{1}, e_{2}, e_{3}\right\}$ of $\mathfrak{g}^{\prime}$ (see [1, $\S 5.3$ (iii)]) such that:

$$
\left[H, e_{1}\right]=\lambda_{1}(H) e_{1}, \quad\left[H, e_{2}\right]=\lambda_{2}(H) e_{2}, \quad\left[H, e_{3}\right]=\lambda_{3}(H) e_{3}
$$

for all $H \in \mathfrak{a}$. Hence $\operatorname{ad}_{H}$ is symmetric for all $H \in \mathfrak{a}$ and its matrix with respect to the basis $\left\{e_{1}, e_{2}, e_{3}\right\}$ is given by

$$
\operatorname{ad}_{H}=\left[\begin{array}{ccc}
\left\langle H, H_{1}\right\rangle & 0 & 0 \\
0 & \left\langle H, H_{2}\right\rangle & 0 \\
0 & 0 & \left\langle H, a H_{1}+b H_{2}\right\rangle
\end{array}\right] .
$$


Let $H_{0}$ be a unit vector in a such that $\left\langle H_{0}, H_{1}\right\rangle=0$ and $\left\langle H_{0}, H_{2}\right\rangle$ $>0$. Observe that $D_{0}=\operatorname{ad}_{H_{0}}$ is positive semidefinite and restricted to $\mathfrak{g}_{2,3}^{\prime}=\mathfrak{g}_{\lambda_{2}}^{\prime} \oplus \mathfrak{g}_{\lambda_{3}}^{\prime}$ is positive definite. Also, $D_{1}=\operatorname{ad}_{H_{1}}$ is positive semidefinite and restricted to $\mathfrak{g}_{1,3}^{\prime}=\mathfrak{g}_{\lambda_{1}}^{\prime} \oplus \mathfrak{g}_{\lambda_{3}}^{\prime}$ is positive defnite. Hence, if $X=c e_{1}+d e_{2}+e e_{3}$ is a unit vector and $Y \in \mathfrak{g}^{\prime}$, it follows from the curvature formula given in Lemma 1.1-(i) that, $\langle R(X, Y) Y, X\rangle=0$ if and only if $\left.P\right|_{\mathfrak{g}_{2,3}^{\prime}} Y$ is proportional to $d e_{2}+e e_{3}$ and $\left.p\right|_{\mathfrak{g}_{1,3}^{\prime}} Y$ is proportional to $c e_{1}+e e_{3}$, where $p$ denotes the orthogonal projection onto the indicated subspaces.

By a simple computation we deduce that if $e \neq 0,\langle R(X, Y) Y, X\rangle$ $=0$ if and only if $Y$ is proportional to $X$. Hence, choosing $d \neq$ $0, e \neq 0$ (or $c \neq 0$ ) for any $Y$ independent of $X$ in $\mathfrak{g}^{\prime}$ we get $\langle R(X, Y) Y, X\rangle<0$. Moreover, for any nonzero vector $H \in \mathfrak{a}$,

$$
\begin{aligned}
\langle R(X, H) H, X\rangle & =-|[H, X]|^{2} \\
& =-c^{2} \lambda_{1}(H)^{2}-d^{2} \lambda_{2}(H)^{2}-e^{2} \lambda_{3}(H)^{2}<0
\end{aligned}
$$

since $\lambda_{2}(H)$ and $\lambda_{3}(H)$ (or $\lambda_{1}(H)$ ) cannot be simultaneously zero. Therefore, if $\gamma$ is the geodesic in $G$ with $\gamma(0)=e, \gamma^{\prime}(0)=X, \gamma$ has rank one and hence $\operatorname{rank}(G)=1$.

Case 2.2. Assume $\mathfrak{g}^{\prime}$ nonabelian. It turns out that either $G$ has rank one or $G$ is an irreducible symmetric space of rank two.

It follows from the lemma that there are three real roots $\lambda_{1}, \lambda_{2}$ and $\lambda_{3}=\lambda_{1}+\lambda_{2}$ with $\lambda_{1}$ and $\lambda_{2}$ independent. Moreover, $\mathfrak{z}$ is the eigenspace associated to $\lambda_{3}$. By the same argument as in Case 2.1 we get an orthonormal basis $\left\{e_{1}, e_{2}, e_{3}\right\}$ of $\mathfrak{g}^{\prime}$ such that $\left[H, e_{i}\right]=$ $\lambda_{i}(H) e_{i}(i=1,2,3)$ for all $H \in \mathfrak{a}$.

Let $H_{i}$ be defined by $\lambda_{i}(H)=\left\langle H, H_{i}\right\rangle(i=1,2), H \in \mathfrak{a}$. We consider a unit vector $H_{0}$ in $\mathfrak{a}$ such that $\left\langle H_{0}, H_{1}+H_{2}\right\rangle=0$ and $\left\langle H_{0}, H_{1}\right\rangle>0$. If $\widetilde{H}=\left(H_{1}+H_{2}\right) /\left|H_{1}+H_{2}\right|$, the matrices of $\operatorname{ad}_{H_{0}}$ and $\operatorname{ad}_{\widetilde{H}}$ with respect to the orthonormal basis $\left\{e_{1}, e_{2}, e_{3}\right\}$ are given by

$$
\begin{aligned}
& \operatorname{ad}_{H_{0}}= {\left[\begin{array}{ccc}
\left\langle H_{0}, H_{1}\right\rangle & & 0 \\
0 & \left\langle H_{0}, H_{2}\right\rangle & 0 \\
0 & 0 & 0
\end{array}\right], } \\
& \operatorname{ad}_{\widetilde{H}}=\left[\begin{array}{ccc}
\left\langle\widetilde{H}, H_{1}\right\rangle & 0 & 0 \\
0 & \left\langle\widetilde{H}, H_{2}\right\rangle & 0 \\
0 & 0 & \left\langle\widetilde{H}, H_{1}+H_{2}\right\rangle
\end{array}\right] .
\end{aligned}
$$

Since $\mathfrak{g}^{\prime}$ is nonabelian, $\left[e_{1}, e_{2}\right]=\varepsilon e_{e}\left(\left[\mathfrak{g}_{\lambda_{1}}^{\prime}, \mathfrak{g}_{\lambda_{2}}^{\prime}\right] \subset \mathfrak{g}_{\lambda_{1}+\lambda_{2}}^{\prime}=\mathfrak{z}\right)$ and we 
may assume that $\varepsilon>0$ (otherwise we change $e_{3}$ to $-e_{3}$ ). Set $e_{4}=$ $H_{0}, e_{5}=\widetilde{H}, \alpha=\left\langle\widetilde{H}, H_{1}\right\rangle, \beta=\left\langle\widetilde{H}, H_{2}\right\rangle$ and $\gamma=\left\langle H_{0}, H_{1}\right\rangle>0$. Then, $\left\{e_{1}, e_{2}, e_{3}, e_{4}, e_{5}\right\}$ is an orthonormal basis of $\mathfrak{g}$ satisfying:

$$
\begin{array}{lll}
{\left[e_{1}, e_{2}\right]=\varepsilon e_{3},} & {\left[e_{1}, e_{3}\right]=0=\left[e_{2}, e_{3}\right],} & \\
{\left[e_{4}, e_{1}\right]=\gamma e_{1},\left[e_{4}, e_{2}\right]=-\gamma e_{2},} & {\left[e_{4}, e_{3}\right]=0=\left[e_{4}, e_{5}\right],} \\
{\left[e_{5}, e_{1}\right]=\alpha e_{1},\left[e_{5}, e_{2}\right]=\beta e_{2},} & {\left[e_{5}, e_{3}\right]=(\alpha+\beta) e_{3}}
\end{array}
$$

with $\varepsilon>0, \gamma>0$ and $\alpha+\beta>0$. Moreover, $\alpha>0$ and $\beta>0$ since $K\left(e_{1}, e_{3}\right)=\frac{1}{4} \varepsilon^{2}-\alpha(\alpha+\beta), K\left(e_{2}, e_{3}\right)=\frac{1}{4} \varepsilon^{2}-\beta(\alpha+\beta)($ see $\S 2,(3))$ and the sectional curvature $K \leq 0$. This special case will be studied in detail in $\S 2$. As we will see, $G$ is isomorphic to the Lie group of $3 \times 3$ upper triangular real matrices of determinant one, and it follows from Corollary 2.8 that $G$ has rank one or two. In the latter case, provided that one multiplies the metric by a suitable positive constant, $G$ is isometric to the irreducible symmetric space of noncompact type and rank two $\mathrm{SL}(3, \mathbf{R}) / \mathrm{SO}(3)$ (see Remark 2.8).

By examining all the cases, Theorem 1.3 follows. Note that $G$ satisfies visibility or not depending on whether $\operatorname{dim} \mathfrak{a}=1$ or 2 .

COROLlARY 1.4. The simply connected homogeneous spaces $H$ of nonpositive curvature, with no flat de Rham factor, with $\operatorname{dim}(H) \leq$ 5 and $\operatorname{rank}(H)=2$ are $H^{2} \times T^{2}, H^{2} \times T^{3}$ or $H$ an irreducible symmetric space of noncompact type.

Proof. It is immediate by Theorem 1.3 and Corollary 4.4 of [6]. $H^{2}, T^{2}$ and $T^{3}$ are as in the statement of Theorem 1.3.

2. Example. Let $\mathfrak{g}$ be the Lie algebra of dimension five generated by $\left\{e_{i}\right\}_{i=1}^{5}$ and Lie bracket given by

$$
\begin{array}{llll}
{\left[e_{1}, e_{2}\right]=\varepsilon e_{3},} & {\left[e_{1}, e_{3}\right]=0=\left[e_{2}, e_{3}\right],} & \\
{\left[e_{4}, e_{1}\right]=\gamma e_{1},} & {\left[e_{4}, e_{2}\right]=-\gamma e_{2},} & {\left[e_{4}, e_{3}\right]=0=\left[e_{4}, e_{5}\right],} \\
{\left[e_{5}, e_{1}\right]=\alpha e_{1},} & {\left[e_{5}, e_{2}\right]=\beta e_{2},} & {\left[e_{5}, e_{3}\right]=(\alpha+\beta) e_{3}}
\end{array}
$$

where $\alpha, \beta, \gamma, \varepsilon$ are positive real numbers. (Note that $\mathfrak{g}^{\prime}$ is spanned by $\left\{e_{1}, e_{2}, e_{3}\right\}$.) We will say that such a $\mathfrak{g}$ is associated to $(\alpha, \beta$, $\gamma, \varepsilon)$.

Let $\langle$,$\rangle be the inner product in \mathfrak{g}$ with respect to which $\left\{e_{i}\right\}_{i=1}^{5}$ is an orthonormal basis of $\mathfrak{g}$, and let $G$ be the simply connected Lie group with Lie algebra $\mathfrak{g}$ and left invariant metric associated to $\langle$,$\rangle .$ By a straightforward computation, using the connection formula and 
the definitions of $R, K$ we get:

$$
\begin{array}{lll}
\nabla_{e_{1}} e_{1}=\gamma e_{4}+\alpha e_{5}, & \nabla_{e_{1}} e_{2}=\frac{1}{2} \varepsilon e_{3}, & \nabla_{e_{1}} e_{3}=-\frac{1}{2} \varepsilon e_{2}, \\
\nabla_{e_{2}} e_{2}=-\gamma e_{4}+\beta e_{5}, & \nabla_{e_{2}} e_{3}=\frac{1}{2} \varepsilon e_{1}, & \nabla_{e_{3}} e_{3}=(\alpha+\beta) e_{5}, \\
\nabla_{e_{1}} e_{4}=-\gamma e_{1}, & \nabla_{e_{1}} e_{5}=-\alpha e_{1}, & \nabla_{e_{2}} e_{4}=\gamma e_{2}, \\
\nabla_{e_{2}} e_{5}=-\beta e_{2}, & \nabla_{e_{3}} e_{4}=0, & \nabla_{e_{3}} e_{5}=-(\alpha+\beta) e_{3} .
\end{array}
$$

$$
\begin{array}{ll}
R\left(e_{1}, e_{2}\right) e_{1}=\left(\frac{3}{4} \varepsilon^{2}+\alpha \beta-\gamma^{2}\right) e_{2}, & R\left(e_{1}, e_{2}\right) e_{2}=\left(\gamma^{2}-\alpha \beta-\frac{3}{4} \varepsilon^{2}\right) e_{1}, \\
R\left(e_{1}, e_{2}\right) e_{3}=-\frac{1}{2} \varepsilon(\alpha+\beta) e_{5}, & R\left(e_{2}, e_{3}\right) e_{1}=\frac{1}{2} \varepsilon\left(\gamma e_{4}+\alpha e_{5}\right), \\
R\left(e_{2}, e_{3}\right) e_{2}=\left(-\frac{1}{4} \varepsilon^{2}+\beta(\alpha+\beta)\right) e_{3}, & R\left(e_{2}, e_{3}\right) e_{3}=\left(\frac{1}{4} \varepsilon^{2}-\beta(\alpha+\beta)\right) e_{2}, \\
R\left(e_{1}, e_{3}\right) e_{1}=\left(-\frac{1}{4} \varepsilon^{2}+\alpha(\alpha+\beta)\right) e_{3}, & R\left(e_{1}, e_{3}\right) e_{2}=\frac{1}{2} \varepsilon\left(\gamma e_{4}-\beta e_{5}\right), \\
R\left(e_{1} e_{3}\right) e_{3}=\left(\frac{1}{4} \varepsilon^{2}-\alpha(\alpha+\beta)\right) e_{1} . &
\end{array}
$$

$$
\begin{array}{ll}
K\left(e_{1}, e_{2}\right)=-\frac{3}{4} \varepsilon^{2}+\gamma^{2}-\alpha \beta, & K\left(e_{1}, e_{3}\right)=\frac{1}{4} \varepsilon^{2}-\alpha(\alpha+\beta), \\
K\left(e_{2}, e_{3}\right)=\frac{1}{4} \varepsilon^{2}-\beta(\alpha+\beta), & K\left(e_{4}, e_{2}\right)=K\left(e_{4}, e_{1}\right)=-\gamma^{2}, \\
K\left(e_{4}, e_{3}\right)=0, & K\left(e_{5}, e_{1}\right)=-\alpha^{2}, \\
K\left(e_{5}, e_{2}\right)=-\beta^{2}, & K\left(e_{5}, e_{3}\right)=-(\alpha+\beta)^{2} .
\end{array}
$$

We note that in all computations above, $\alpha, \beta, \gamma$ and $\varepsilon$ may be arbitrary.

(4) We remark that it will be shown in $\S 3.1$ that if $\alpha=\beta=\varepsilon / 2=$ $\gamma / \sqrt{ } 3$ then $G$ is a symmetric space.

Conversely, assuming $G$ symmetric (i.e., $\nabla R=0$ ) we get $\alpha=$ $\beta=\varepsilon / 2=\gamma / \sqrt{ } 3$. This follows by a straightforward computation of $\nabla_{e_{1}}\left(R\left(e_{1}, e_{2}\right) e_{1}\right), \nabla_{e_{2}}\left(R\left(e_{1}, e_{2}\right) e_{3}\right)$ and $\nabla_{e_{1}}\left(R\left(e_{1}, e_{2}\right) e_{4}\right)$ using $\nabla R=$ 0 and (1) and (2) above.

The following lemma is proved in [7]. We state it here since it is applied in Lemma 2.2 to obtain an expression for the sectional curvature that will be used repeatedly.

LEMMA 2.1. Let $\mathfrak{g}$ be a solvable Lie algebra with an inner product $\langle$,$\rangle such that \mathfrak{a}$, the orthogonal complement of $\mathfrak{g}^{\prime}$ is abelian. If $\left.\operatorname{ad}_{H}\right|_{\mathfrak{g}^{\prime}}$ is symmetric with respect to $\langle$,$\rangle for all H \in \mathfrak{a}$, then

$$
\begin{aligned}
\langle R(X+ & H, Y+T)(Y+T), X+H\rangle \\
= & \langle R(X, Y) Y, X\rangle-|[H, Y]-[T, X]|^{2} \\
& -\langle[H, Y]-[T, X],[X, Y]\rangle+\langle[[H, Y]-[T, X], X], Y\rangle \\
& -\langle[[H, Y]-[T, X], Y], X\rangle
\end{aligned}
$$

for all $X, Y \in \mathfrak{g}^{\prime}$ and $H, T \in \mathfrak{a}$. 
LemMA 2.2. Let $a, b, c, r, s, t$ be real numbers and $H, T$ elements in $\mathfrak{a}$; then

$$
\begin{gathered}
\left\langle R\left(a e_{1}+b e_{2}+c e_{3}+H, r e_{1}+s e_{2}+t e_{3}+T\right)\left(r e_{1}+s e_{2}+t e_{3}+T\right)\right. \\
\left.a e_{1}+b e_{2}+c e_{3}+H\right\rangle \\
=\left[(c s-b t)^{2} K\left(e_{2}, e_{3}\right)+\varepsilon(c s-b t)\left(a \lambda_{1}(T)-r \lambda_{1}(H)\right)\right. \\
\left.-\left(a \lambda_{1}(T)-r \lambda_{1}(H)\right)^{2}\right] \\
+\left[(a t-c r)^{2} K\left(e_{1}, e_{3}\right)+\varepsilon(a t-c r)\left(b \lambda_{2}(T)-s \lambda_{2}(H)\right)\right. \\
\left.-\left(b \lambda_{2}(T)-s \lambda_{2}(H)\right)^{2}\right] \\
+\left[(a s-b r)^{2} K\left(e_{1}, e_{2}\right)+\varepsilon(a s-b r)\left(c \lambda_{3}(T)-t \lambda_{3}(H)\right)\right. \\
\left.-\left(c \lambda_{3}(T)-t \lambda_{3}(H)\right)^{2}\right]
\end{gathered}
$$

where $\lambda_{i}(i=1,2,3)$ are defined by

$$
\begin{gathered}
\lambda_{1}(U)=\left\langle U, \gamma e_{4}+\alpha e_{5}\right\rangle, \quad \lambda_{2}(U)=\left\langle U,-\gamma e_{4}+\beta e_{5}\right\rangle \text { and } \\
\lambda_{3}(U)=\left(\lambda_{1}+\lambda_{2}\right)(U)=(\alpha+\beta)\left\langle U, e_{5}\right\rangle \text { for all } U \in \mathfrak{a} .
\end{gathered}
$$

Proof. First of all we show that,

$$
\begin{aligned}
\left\langleR \left( a e_{1}+\right.\right. & \left.\left.b e_{2}+c e_{3}, r e_{1}+s e_{2}+t e_{3}\right)\left(r e_{1}+s e_{2}+t e_{3}\right), a e_{1}+b e_{2}+c e_{3}\right\rangle \\
= & (a s-b r)^{2} K\left(e_{1}, e_{2}\right)+t^{2}\left(a^{2} K\left(e_{1}, e_{3}\right)+b^{2} K\left(e_{2}, e_{3}\right)\right) \\
& +c^{2}\left(r^{2} K\left(e_{1}, e_{3}\right)+s^{2} K\left(e_{2}, e_{3}\right)\right) \\
& -2 c t\left(a r K\left(e_{1}, e_{3}\right)+b s K\left(e_{2}, e_{3}\right)\right) .
\end{aligned}
$$

Let $X=a e_{1}+b e_{2}$ and $Y=r e_{1}+s e_{2}$. Applying the linearity of $R$ and using that $R(X, Y) e_{3}$ is an element in a (see (2)) we have,

$$
\begin{aligned}
\langle R(X & \left.\left.+c e_{3}, Y+t e_{3}\right)\left(Y+t e_{3}\right), X+c e_{3}\right\rangle \\
= & \langle R(X, Y) Y, X\rangle+2 c t\left\langle R\left(X, e_{3}\right) Y, e_{3}\right\rangle+t^{2}\left\langle R\left(X, e_{3}\right) e_{3}, X\right\rangle \\
& +c^{2}\left\langle R\left(e_{3}, Y\right) Y, e_{3}\right\rangle .
\end{aligned}
$$

Now, since $R\left(e_{1}, e_{3}\right) e_{3}$ is a multiple of $e_{1}$ (see (2)), an easy calculation shows that

(ii) $\left\langle R\left(X, e_{3}\right) e_{3}, Y\right\rangle=\operatorname{ar} K\left(e_{1}, e_{3}\right)+b s K\left(e_{2}, e_{3}\right)$.

Hence, (i) is deduced from (ii) and the equality

$$
\langle R(X, Y) Y, X\rangle=|X \wedge Y|^{2} K\left(e_{1}, e_{2}\right)=(a s-b r)^{2} K\left(e_{1}, e_{2}\right) .
$$

Now, the formula stated in the lemma follows by a straightforward computation using Lemma 2.1 .

Next, in the two propositions below we find necessary and sufficient conditions for $G$ to have nonpositive curvature in terms of $\alpha, \beta, \gamma, \varepsilon$. 
Proposition 2.3. If $G$ has sectional curvature $K \leq 0$ then the following relations among $\alpha, \beta, \gamma, \varepsilon$ hold: $\varepsilon^{2} \leq 2 \beta(\alpha+\beta), \varepsilon^{2} \leq$ $2 \alpha(\alpha+\beta), \gamma^{2} \leq \frac{1}{2} \varepsilon^{2}+\alpha \beta$. In particular, $K\left(e_{2}, e_{3}\right), K\left(e_{1}, e_{3}\right)$ and $K\left(e_{1}, e_{2}\right)$ are all strictly negative.

Proof. We first show that if $\varepsilon^{2}-2 \beta(\alpha+\beta)>0$ (or $\varepsilon^{2}-2 \alpha(\alpha+\beta)>0$ ) then there exists a plane $\pi$ in $\mathfrak{g}$ with sectional curvature $K(\pi)>0$. In fact, if we take $H=0, T=\lambda e_{4}$ we have $\lambda_{3}=\lambda_{3}(T)=0$ and $\lambda_{1}=\lambda_{1}(T)=-\gamma \lambda$ with $\lambda_{1} \neq 0$ for any nonzero real $\lambda$. Hence, by applying the curvature formula given by Lemma 2.2 , we get

$$
\begin{aligned}
& \left\langle R\left(a e_{1}+c e_{3}, e_{2}+\lambda e_{4}\right)\left(e_{2}+\lambda e_{4}\right), a e_{1}+c e_{3}\right\rangle \\
& \quad=c^{2} K\left(e_{2}, e_{3}\right)+a^{2}\left(K\left(e_{1}, e_{2}\right)-\lambda_{1}^{2}\right)-\varepsilon \lambda_{1} a c,
\end{aligned}
$$

for any real numbers $a, c$. If we consider this expression as a polynomial of second degree in $a\left(K \leq 0, \lambda_{1} \neq 0\right)$ its discriminant $\Delta$ is given by

$$
\Delta=c^{2}\left(\lambda_{1}^{2}\left(\varepsilon^{2}+4 K\left(e_{2}, e_{3}\right)\right)-4 K\left(e_{1}, e_{2}\right) K\left(e_{2}, e_{3}\right)\right) .
$$

Note that $\varepsilon^{2}+4 K\left(e_{2}, e_{3}\right)=2\left(\varepsilon^{2}-2 \beta(\alpha+\beta)\right)$. Thus, by choosing $\lambda$ so that

$$
\lambda^{2} \gamma^{2}=\lambda_{1}^{2}>\frac{4 K\left(e_{2}, e_{3}\right) K\left(e_{1}, e_{2}\right)}{2\left(\varepsilon^{2}-2 \beta(\alpha+\beta)\right)}
$$

we get $\Delta$ strictly positive for any nonzero real $c$. For this $\lambda$ and nonzero $c$, a real number $a$ can be chosen satisfying

$$
K\left(a e_{1}+c e_{3}, e_{2}+\lambda e_{4}\right)>0 .
$$

The other statement follows in the same way by interchanging the roles of $e_{1}$ and $e_{2}$. Hence, the first two inequalities follow.

Now we prove the last one. In the same way as above, if we take $T=\lambda\left(-\beta e_{4}+\gamma e_{5}\right)$ with $\lambda \neq 0$ (hence, $\lambda_{2}=\lambda_{2}(T)=0$ and $\lambda_{3}=$ $\left.\lambda_{3}(T)=\lambda \gamma(\alpha+\beta) \neq 0\right)$ and applying the curvature formula again, we have

$$
\begin{aligned}
& \left\langle R\left(b e_{2}+c e_{3}, e_{1}+T\right)\left(e_{1}+T\right), b e_{2}+c e_{3}\right\rangle \\
& \quad=b^{2} K\left(e_{1}, e_{2}\right)+c^{2}\left(K\left(e_{1}, e_{3}\right)-\lambda_{3}^{2}\right)-\varepsilon \lambda_{3} b c,
\end{aligned}
$$

which considered as a polynomial (of second degree) in $c$ has discriminant

$$
\Delta=b^{2}\left(\lambda_{3}^{2}\left(\varepsilon^{2}+4 K\left(e_{1}, e_{2}\right)\right)-4 K\left(e_{1}, e_{2}\right) K\left(e_{1}, e_{3}\right)\right) .
$$


Note firstly that $\varepsilon^{2}+4 K\left(e_{1}, e_{2}\right)=2\left(-\varepsilon^{2}+2\left(\gamma^{2}-\alpha \beta\right)\right)$. Thus, if we assume $2\left(\gamma^{2}-\alpha \beta\right)-\varepsilon^{2}>0$ (or $\left.\gamma^{2}>\varepsilon^{2} / 2+\alpha \beta\right)$, taking $\lambda$ in such a way that

$$
\lambda^{2} \gamma^{2}(\alpha+\beta)^{2}=\lambda_{3}^{2}>\frac{4 K\left(e_{1}, e_{2}\right) K\left(e_{1}, e_{3}\right)}{\varepsilon^{2}+4 K\left(e_{1}, e_{2}\right)},
$$

for any nonzero real $b$ we get $\Delta>0$. Hence, a real $c$ can be chosen such that $K\left(b e_{2}+c e_{3}, e_{1}+T\right)>0$. The assertion follows since $K \leq 0$.

Proposition 2.4. The conditions $\varepsilon^{2} \leq 2 \beta(\alpha+\beta), \varepsilon^{2} \leq 2 \alpha(\alpha+\beta)$, $\gamma^{2} \leq \frac{1}{2} \varepsilon^{2}+\alpha \beta$ are sufficient for $G$ to have sectional curvature $K \leq 0$.

Proof. We note from the curvature formula given in Lemma 2.2 that each term in between brackets is a polynomial of second degree $\left(K\left(e_{1}, e_{3}\right), K\left(e_{2}, e_{3}\right)\right.$ and $K\left(e_{1}, e_{2}\right)$ are negative) in $(c s-b t)$, $(a t-c r)$ and $(a s-b r)$ respectively, with discriminant

$$
\begin{array}{lll}
\left(a \lambda_{1}(T)-r \lambda_{1}(H)\right)^{2} & \left(\varepsilon^{2}+4 K\left(e_{2}, e_{3}\right)\right), & \\
\left(b \lambda_{2}(T)-s \lambda_{2}(H)\right)^{2} & \left(\varepsilon^{2}+4 K\left(e_{1}, e_{3}\right)\right), \quad \text { and } \\
\left(c \lambda_{3}(T)-t \lambda_{3}(H)\right)^{2} & \left(\varepsilon^{2}+4 K\left(e_{1}, e_{2}\right)\right) .
\end{array}
$$

Under our assumption, $\varepsilon^{2} \leq 2 \beta(\alpha+\beta), \varepsilon^{2} \leq 2 \alpha(\alpha+\beta)$ and $\gamma^{2} \leq \frac{1}{2} \varepsilon^{2}+\alpha \beta$, these discriminants are nonpositive and therefore each polynomial is also nonpositive. Thus,

$$
K\left(a e_{1}+b e_{2}+c e_{3}+H, r e_{1}+s e_{2}+t e_{3}+T\right) \leq 0
$$

for any real $a, b, c, r, s, t$ and $H, T \in \mathfrak{a}$. Hence, $K \leq 0$.

Next, under the assumption $K \leq 0$, we will get some conditions for $G$ to have rank one.

Proposition 2.5. The real number $\varepsilon$ must satisfy $\varepsilon \leq \alpha+\beta$. Moreover, $G$ has rank one if $\varepsilon<\alpha+\beta$.

Proof. The condition $\varepsilon \leq \alpha+\beta$ follows immediately from the first two inequalities of Proposition 2.3. We note that $(\alpha+\beta)^{2} \leq 2 \alpha(\alpha+\beta)$ or $(\alpha+\beta)^{2} \leq 2 \beta(\alpha+\beta)$ depending on whether $\beta \leq \alpha$ or $\alpha \leq \beta$ respectively. Consequently, $\varepsilon<\alpha+\beta$ if and only if $\varepsilon^{2}<2 \alpha(\alpha+\beta)$ or $\varepsilon^{2}<2 \beta(\alpha+\beta)$. 
Next we check the last statement. Using Lemma 2.2, for each $Y \in \mathfrak{g}^{\prime}$ orthogonal to $e_{3}$ and $T \in \mathfrak{a}$, we have

$$
\begin{gathered}
\left\langle R\left(e_{1}+e_{2}, Y+t e_{3}+T\right)\left(Y+t e_{3}+T\right), e_{1}+e_{2}\right\rangle \\
=t^{2}\left(K\left(e_{1}, e_{3}\right)+K\left(e_{2}, e_{3}\right)\right)-t \varepsilon\left(\lambda_{1}-\lambda_{2}\right) \\
-\lambda_{1}^{2}-\lambda_{2}^{2}+\left|\left(e_{1}+e_{2}\right) \wedge Y\right|^{2} K\left(e_{1}, e_{2}\right) .
\end{gathered}
$$

This expression is a polynomial $p(t)$ of degree two in $t$ whose discriminant $\Delta$ is given by

$$
\begin{aligned}
\Delta= & \varepsilon^{2}\left(\lambda_{1}-\lambda_{2}\right)^{2} \\
& +4\left(K\left(e_{1}, e_{3}\right)+K\left(e_{2}, e_{3}\right)\right)\left(\lambda_{1}^{2}+\lambda_{2}^{2}-\left|\left(e_{1}+e_{2}\right) \wedge Y\right|^{2} K\left(e_{1}, e_{2}\right)\right) .
\end{aligned}
$$

Now, we assume $\varepsilon<\alpha+\beta$. Since $K \leq 0$ we have

$$
\Delta \leq \varepsilon^{2}\left(\lambda_{1}-\lambda_{2}\right)^{2}+4\left(K\left(e_{1}, e_{3}\right)+K\left(e_{2}, e_{3}\right)\right)\left(\lambda_{1}^{2}+\lambda_{2}^{2}\right) .
$$

If we substitute the expressions for $K\left(e_{1}, e_{3}\right)$ and $K\left(e_{2}, e_{3}\right)$ into the expression above, we get

$$
\begin{aligned}
\Delta & \leq \varepsilon^{2}\left(\lambda_{1}-\lambda_{2}\right)^{2}+4\left(\frac{1}{2} \varepsilon^{2}-(\alpha+\beta)^{2}\right)\left(\lambda_{1}^{2}+\lambda_{2}^{2}\right) \\
& =\varepsilon^{2}\left(\lambda_{1}-\lambda_{2}\right)^{2}+2 \varepsilon^{2}\left(\lambda_{1}^{2}+\lambda_{2}^{2}\right)-4(\alpha+\beta)^{2}\left(\lambda_{1}^{2}+\lambda_{2}^{2}\right) \\
& =\varepsilon^{2}\left(3 \lambda_{1}^{2}+3 \lambda_{2}^{2}-2 \lambda_{1} \lambda_{2}\right)-4(\alpha+\beta)^{2}\left(\lambda_{1}^{2}+\lambda_{2}^{2}\right) .
\end{aligned}
$$

Now, we consider the two cases, $T \neq 0$ and $T=0$. If $T \neq 0$, since $\lambda_{1}(T)$ and $\lambda_{2}(T)$ are not simultaneously zero, $3 \lambda_{1}^{2}+3 \lambda_{2}^{2}-2 \lambda_{1} \lambda_{2}>$ $\left(\lambda_{1}-\lambda_{2}\right)^{2} \geq 0$. Hence, if $\varepsilon<\alpha+\beta$, we get

$$
\begin{aligned}
\Delta & <(\alpha+\beta)^{2}\left(3 \lambda_{1}^{2}+3 \lambda_{2}^{2}-2 \lambda_{1} \lambda_{2}\right)-4(\alpha+\beta)^{2}\left(\lambda_{1}^{2}+\lambda_{2}^{2}\right) \\
& =-(\alpha+\beta)^{2}\left(\lambda_{1}+\lambda_{2}\right)^{2} \leq 0,
\end{aligned}
$$

and then $p(t)<0$ for all real $t, T \neq 0$ in $\mathfrak{a}$ and $Y$ in $\mathfrak{g}^{\prime}$ orthogonal to $e_{3}$.

If $T=0, p(t)=t^{2}\left(K\left(e_{1}, e_{3}\right)+K\left(e_{2}, e_{3}\right)\right)+\left|\left(e_{1}+e_{2}\right) \wedge Y\right|^{2} K\left(e_{1}, e_{2}\right)<$ 0 whenever $t \neq 0$ or $Y$, orthogonal to $e_{3}$, is independent of $e_{1}+e_{2}$. (Note that $K\left(e_{1}+e_{2}, Y\right)=K\left(e_{1}, e_{2}\right)<0$.)

Therefore, $K\left(e_{1}+e_{2}, Y+t e_{3}+T\right)<0$ for all real number $t, T \in \mathfrak{a}$, $Y \in \mathfrak{g}^{\prime}$ orthogonal to $e_{3}$ and independent of $e_{1}+e_{2}$. Thus, the geodesic $\gamma$ in $G$ satisfying $\gamma(0)=e$ and $\gamma^{\prime}(0)=e_{1}+e_{2}$ has rank one.

Proposition 2.6. The numbers $\alpha, \beta, \gamma$ satisfy the inequalities $\gamma^{2}-$ $2 \alpha \beta-\beta^{2} \leq 0$ and $\gamma^{2}-2 \alpha \beta-\alpha^{2} \leq 0$. Moreover, if $\gamma^{2}-2 \alpha \beta-\beta^{2}<0$ or $\gamma^{2}-2 \alpha \beta-\alpha^{2}<0, G$ has rank one.

Proof. The first two inequalities follow immediately from Proposition $2.3\left(\gamma^{2}-\alpha \beta \leq \varepsilon^{2} / 2\right)$. 
Now, we will show the last assertion. Applying Lemma 2.2, for each $T \in \mathfrak{a}$ and $Y$ in $\mathfrak{g}^{\prime}$ orthogonal to $e_{2}$, we have

$$
\begin{aligned}
\left\langleR \left( e_{1}\right.\right. & \left.\left.+e_{3}, Y+s e_{2}+T\right)\left(Y+s e_{2}+T\right), e_{1}+e_{3}\right\rangle \\
= & s^{2}\left(K\left(e_{1}, e_{2}\right)+K\left(e_{2}, e_{3}\right)\right)+s \varepsilon\left(\lambda_{1}+\lambda_{3}\right)-\lambda_{1}^{2}-\lambda_{3}^{2} \\
& +\left|\left(e_{1}+e_{3}\right) \wedge Y\right|^{2} K\left(e_{1}, e_{3}\right)=p(s),
\end{aligned}
$$

where $\lambda_{i}=\lambda_{i}(T)(i=1,3)$ are defined as in Lemma 2.2.

Note that $p(s)$ is a polynomial of degree two in $s$ whose discriminant $\Delta$ is given by

$$
\begin{aligned}
\Delta= & \varepsilon^{2}\left(\lambda_{1}+\lambda_{3}\right)^{2}+4\left(K\left(e_{1}, e_{2}\right)+K\left(e_{2}, e_{3}\right)\right) \\
& \times\left(\lambda_{1}^{2}+\lambda_{3}^{2}-\left|\left(e_{1}+e_{3}\right) \wedge Y\right|^{2} K\left(e_{1}, e_{3}\right)\right) .
\end{aligned}
$$

Substituting $K\left(e_{1}, e_{2}\right)$ and $K\left(e_{2}, e_{3}\right)$ for its expressions, and since $K \leq 0$ we get,

$$
\begin{aligned}
\Delta & \leq \varepsilon^{2}\left(\lambda_{1}+\lambda_{3}\right)^{2}+4\left(-\frac{1}{2} \varepsilon^{2}+\gamma^{2}-2 \alpha \beta-\beta^{2}\right)\left(\lambda_{1}^{2}+\lambda_{3}^{2}\right) \\
& =\varepsilon^{2}\left(\lambda_{1}+\lambda_{3}\right)^{2}-2 \varepsilon^{2}\left(\lambda_{1}^{2}+\lambda_{3}^{2}\right)+4\left(\gamma^{2}-2 \alpha \beta-\beta^{2}\right)\left(\lambda_{1}^{2}+\lambda_{3}^{2}\right) \\
& =-\varepsilon^{2}\left(\lambda_{1}-\lambda_{3}\right)^{2}+4\left(\gamma^{2}-2 \alpha \beta-\beta^{2}\right)\left(\lambda_{1}^{2}+\lambda_{3}^{2}\right) .
\end{aligned}
$$

To prove that $G$ has rank one we will see that if $\gamma^{2}-2 \alpha \beta-\beta^{2}<0$ then $K\left(e_{1}+e_{3}, Y+s e_{2}+T\right)<0$ for all $s, T$ in $\mathfrak{a}, Y \in \mathfrak{g}^{\prime}$ orthogonal to $e_{2}$ and independent of $e_{1}+e_{3}$. We first consider the case $T \neq 0$; since $\lambda_{1}(T) \neq 0$ we have $\Delta<-\varepsilon^{2}\left(\lambda_{1}-\lambda_{2}\right)^{2} \leq 0$ and hence, the polynomial $p$ satisfies $p(s)<0$ for all $s, T \neq 0$ in $\mathfrak{a}$ and $Y \in \mathfrak{g}^{\prime}$ orthogonal to $e_{3}$. If $T=0$,

$$
p(s)=s^{2}\left(K\left(e_{1}, e_{2}\right)+K\left(e_{2}, e_{3}\right)\right)+\left|\left(e_{1}+e_{3}\right) \wedge Y\right|^{2} K\left(e_{1}, e_{3}\right)<0
$$

whenever $s \neq 0$ or $Y \in \mathfrak{g}^{\prime}$, orthogonal to $e_{3}$, is independent of $e_{1}+e_{3}\left(K\left(e_{1}, e_{3}\right)<0\right.$ and $\left.K\left(e_{2}, e_{3}\right)<0\right)$. Therefore, the assertion is proved and consequently, the geodesic $\gamma$ in $G$ such that $\gamma(0)=e$ and $\gamma^{\prime}(0)=e_{1}+e_{3}$ has rank one.

If $\gamma^{2}-2 \alpha \beta-\alpha^{2}<0$, interchanging the roles of $e_{1}$ and $e_{2}$, we also obtain that $G$ has rank one.

We summarize the preceding results in the following:

THEOREM 2.7. Let $G$ be the simply connected Lie group with Lie algebra associated to $(\alpha, \beta, \gamma, \varepsilon)$ and left invariant metric as defined above. Then $G$ has sectional curvature $K \leq 0$ if and only if

$$
\varepsilon^{2} \leq 2 \alpha(\alpha+\beta), \quad \varepsilon^{2} \leq 2 \beta(\alpha+\beta) \quad \text { and } \quad \gamma^{2} \leq \frac{\varepsilon^{2}}{2}+\alpha \beta
$$


Moreover, $G$ has rank one if any of the following conditions hold:

$$
\varepsilon<\alpha+\beta, \quad \gamma^{2}-2 \alpha \beta-\alpha^{2}<0, \quad \gamma^{2}-2 \alpha \beta-\beta^{2}<0 .
$$

COROLlaRy 2.8. If $G$ has nonpositive curvature, then $G$ has rank one or two and in the latter case, $\alpha=\beta=\varepsilon / 2=\gamma / \sqrt{ } 3$.

Proof. We note first that the roots of $\mathfrak{a}$ in $\mathfrak{g}^{\prime}$ are given by $\lambda_{1}(H)=$ $\left\langle H, \gamma e_{4}+\alpha e_{5}\right\rangle, \lambda_{2}(H)=\left\langle H,-\gamma e_{4}+\beta e_{5}\right\rangle, \lambda_{3}=\lambda_{1}+\lambda_{2}$ for all $H \in \mathfrak{a}$, where $\lambda_{1}$ and $\lambda_{2}$ are independent with associated root spaces $\mathfrak{g}_{\lambda_{i}}^{\prime}=$ $\boldsymbol{R} e_{i}(i=1,2,3)$. Thus, $\mathfrak{g}_{0}^{\prime}=0=\mathfrak{a}_{0}$ and hence $G$ has no de Rham flat factor. Then, it follows from Theorem 1.3 of [7] that $G$ has rank one or two. If $\operatorname{rank}(G)=2$, Theorem 2.7 implies that $\varepsilon=\alpha+\beta$ and $\gamma^{2}-2 \alpha \beta-\beta^{2}=0=\gamma^{2}-2 \alpha \beta-\alpha^{2}$. Hence, $\alpha=\beta=\varepsilon / 2=\gamma / \sqrt{ } 3$.

REMARK 2.8. It will be shown in $\S 3$ (3.1) that when $\alpha=\beta=\varepsilon / 2=$ $\gamma / \sqrt{ } 3, G$ coincides with the symmetric space $\operatorname{SL}(3, \mathbf{R}) / \mathrm{SO}(3)$, provided we multiply the metric by a suitable positive constant.

\section{The group of $3 \times 3$ upper triangular real matrices of determinant one.}

3.1. Let $G$ be the solvable simply connected Lie group of $3 \times 3$ upper triangular real matrices of determinant one. Its Lie algebra $\mathfrak{g}$ consists of the $3 \times 3$-upper triangular real matrices having trace zero and has a basis $\left\{E_{i}\right\}_{i=1}^{5}$ given by

$$
\begin{aligned}
& E_{1}=\left[\begin{array}{lll}
0 & 1 & 0 \\
0 & 0 & 0 \\
0 & 0 & 0
\end{array}\right], \quad E_{2}=\left[\begin{array}{lll}
0 & 0 & 0 \\
0 & 0 & 1 \\
0 & 0 & 0
\end{array}\right], \quad E_{3}=\left[\begin{array}{lll}
0 & 0 & 1 \\
0 & 0 & 0 \\
0 & 0 & 0
\end{array}\right], \\
& E_{4}=\left[\begin{array}{rrr}
1 & 0 & 0 \\
0 & -2 & 0 \\
0 & 0 & 1
\end{array}\right] \text { and } E_{5}=\frac{1}{3}\left(E_{5}^{1}+E_{5}^{2}\right), \text { where } \\
& E_{5}^{1}=\left[\begin{array}{rrr}
1 & 0 & 0 \\
0 & 1 & 0 \\
0 & 0 & -2
\end{array}\right] \text { and } E_{5}^{2}=\left[\begin{array}{rrr}
2 & 0 & 0 \\
0 & -1 & 0 \\
0 & 0 & -1
\end{array}\right] .
\end{aligned}
$$

Let $\alpha, \beta, \gamma, \varepsilon$ be any positive real numbers. Setting $e_{1}=2 \alpha E_{1}$, $e_{2}=2 \beta E_{2}, e_{3}=(4 \alpha \beta / \varepsilon) E_{3}, e_{4}=\gamma / 3 E_{4}$ and $e_{5}=\frac{1}{3}\left(\beta E_{5}^{1}+\alpha E_{5}^{2}\right)$, 
we obtain a basis $\left\{e_{i}\right\}_{i=1}^{5}$ of $\mathfrak{g}$ satisfying:

$$
\begin{array}{lll}
{\left[e_{1}, e_{2}\right]=\varepsilon e_{3},} & {\left[e_{1}, e_{3}\right]=0=\left[e_{2}, e_{3}\right],} & \\
{\left[e_{4}, e_{1}\right]=\gamma e_{1},} & {\left[e_{4}, e_{2}\right]=-\gamma e_{2},} & {\left[e_{4}, e_{3}\right]=0=\left[e_{4}, e_{5}\right],} \\
{\left[e_{5}, e_{1}\right]=\alpha e_{1},} & {\left[e_{5}, e_{2}\right]=\beta e_{2},} & {\left[e_{5}, e_{3}\right]=(\alpha+\beta) e_{3} .}
\end{array}
$$

That is, $\mathfrak{g}$ is isomorphic, as a Lie algebra of matrices, to the Lie algebra associated to $(\alpha, \beta, \gamma, \varepsilon)$ which was studied in $\S 2$. I learned of this realization from [9]. Thus, considering on $\mathfrak{g}$ the inner product $\langle$,$\rangle such that \left\{e_{i}\right\}_{i=1}^{5}$ is an orthonormal basis of $\mathfrak{g}$, we see that any choice of $(\alpha, \beta, \gamma, \varepsilon)$ gives us a left invariant metric on $G$. Moreover, almost all these metrics are not isometric. Note, since $\mathfrak{g}^{\prime}$ is nonabelian, it is deduced from the proof of Theorem 1.3 that any left invariant metric on $G$ of $K \leq 0$ is, up to an isometry, the metric associated to some $(\alpha, \beta, \gamma, \varepsilon)$.

In the case $\alpha=\beta=\varepsilon / 2$ and $\gamma=(\sqrt{ } 3 / 2) \varepsilon$, provided that we multiply the metric by a suitable positive constant, $G$ is isometric to the irreducible symmetric space of noncompact type and rank two $H=\mathrm{SL}(3, \mathbf{R}) / \mathrm{SO}(3)$. In fact, $G=N A$ where $N=\exp \mathfrak{n}, \mathfrak{n}$ is the Lie algebra of $3 \times 3$-strictly upper triangular real matrices and $A$ is the group of diagonal real matrices of determinant one. Since $\operatorname{SL}(3, \mathbf{R})=$ $\mathrm{SO}(3) N A$ is an Iwasawa decomposition for $\mathrm{SL}(3, \mathbf{R})$, it is well known (see [1, Lemma 2.4] and [10]) that $G$ acts simply transitively on $H$. Now, if $\mathfrak{p}$ is the orthogonal complement of $\mathfrak{s o}(3)$ in $\mathfrak{s l}(3, \mathbf{R})$ with respect to the Killing form $B$ on $\mathfrak{s l}(3, \mathbf{R})(B(X, Y)=6 \operatorname{tr}(X, Y)), \mathfrak{p}$ may be identified with the tangent space to $H$ at $o=\operatorname{ISO}(3)$, and the metric on $T_{0} H$ corresponds to the restriction of the Killing form to $\mathfrak{p}$. If $\theta$ is the Cartan involution in $\mathfrak{s l}(3, \mathbf{R})$ relative to $\mathfrak{s o}(3)(\theta(X)=$ $\left.-X^{t}\right)$ then the inner product in $\mathfrak{g}=\mathfrak{n} \oplus \mathfrak{a}$, where $\mathfrak{a}$ is the Lie algebra of $A$, obtained from the metric on $\mathfrak{p}$ is given by

$$
(X+H, Y+T)=-\frac{1}{2} B(X, \theta Y)+B(H, T) \quad \text { for } X, Y \in \mathfrak{n}, H, T \in \mathfrak{a} .
$$

It is a straightforward computation to see that the metric given by $\alpha=\beta, \varepsilon=2 \alpha$ and $\gamma^{2}=3 \alpha^{2}$ (that is, $\left\langle E_{i}, E_{j}\right\rangle=0, i \neq j$, $\left.\left|E_{1}\right|^{2}=\left|E_{2}\right|^{2}=\left|E_{3}\right|^{2}=1 / 4 \alpha^{2},\left|E_{4}\right|^{2}=3 / \alpha^{2},\left|E_{5}\right|^{2}=1 / \alpha^{2}\right)$ is a multiple of the metric $($,$) . Moreover, ()=,12 \alpha^{2}\langle$,$\rangle .$

3.2. Next we will obtain a comparison result between the symmetric metric on $G$ and nonsymmetric metrics. The idea is to compare the 
curvature associated to the 4-tuples $(\alpha, \beta, \gamma, \varepsilon)$ and $\left(\alpha_{0}, \alpha_{0}, \sqrt{ } 3 \alpha_{0}\right.$, $\left.2 \alpha_{0}\right)$ where the last one corresponds to the symmetric case.

Let $\alpha, \beta, \gamma, \varepsilon$ be positive real numbers and let $\left\{E_{i}\right\}_{i=1}^{5}$ and $\left\{e_{i}\right\}_{i=1}^{5}$ be as in (3.1). We consider the inner product $\langle$,$\rangle on \mathfrak{g}$ such that $\left\{e_{i}\right\}_{i=1}^{5}$ is an orthonormal basis of $\mathfrak{g}$. Then we have:

$$
\begin{aligned}
\left\langle E_{i}, E_{j}\right\rangle & =0, \quad i \neq j, \\
\left|E_{1}\right|^{2}=\frac{1}{4 \alpha^{2}}, \quad\left|E_{2}\right|^{2}=\frac{1}{4 \beta^{2}}, \quad\left|E_{3}\right|^{2} & =\frac{\varepsilon^{2}}{16 \alpha^{2} \beta^{2}}, \\
\left|E_{4}\right|^{2}=\frac{9}{\gamma^{2}}, \quad\left|\beta E_{5}^{1}+\alpha E_{5}^{2}\right|^{2} & =9 .
\end{aligned}
$$

In order to compare the metrics associated to different $(\alpha, \beta, \gamma, \varepsilon)$ it is convenient to multiply the metric \langle\rangle by the factor $4 \alpha^{2} \beta^{2} / \varepsilon^{2}$. Then the orthonormal basis with respect to the new metric, that we also denote by $\left\{e_{i}\right\}$ and $\langle$,$\rangle is given by$

$$
\begin{array}{ll}
e_{1}=\frac{\varepsilon}{\beta} E_{1}, & e_{2}=\frac{\varepsilon}{\alpha} E_{2}, \quad e_{3}=2 E_{3}, \\
e_{4}=\frac{\varepsilon \gamma}{6 \alpha \beta} E_{4}, & e_{5}=\frac{\varepsilon}{6 \alpha \beta}\left(\alpha E_{5}^{2}+\beta E_{5}^{1}\right) .
\end{array}
$$

Now, observe that the metric on $\mathfrak{z}=\mathbf{R} E_{3}$, the center of $\mathfrak{g}^{\prime}$, does not depend on $(\alpha, \beta, \gamma, \varepsilon)$; that is, if $Z_{1}, Z_{2} \in \mathfrak{z}$ then $\left\langle Z_{1}, Z_{2}\right\rangle=$ $\left\langle Z_{1}, Z_{2}\right\rangle_{0}$ where $\langle,\rangle_{0}$ is the metric associated to $\left(\alpha_{0}, \beta_{0}, \gamma_{0}, \varepsilon_{0}\right)$. Therefore, since $\left[\mathfrak{g}^{\prime}, \mathfrak{g}^{\prime}\right]=\left[\mathfrak{z}^{\perp}, \mathfrak{z}^{\perp}\right] \subset \mathfrak{z}\left(\mathfrak{z}^{\perp}\right.$ is the orthogonal complement of $\mathfrak{z}$ in $\mathfrak{g}^{\prime}$ ), for $X, Y \in \mathfrak{g}^{\prime}$ and $H, T \in \mathfrak{a}$, the curvature formula given in Lemma 2.1 tells us that the last three terms of its expression do not depend on $(\alpha, \beta, \gamma, \varepsilon)$.

Let $X=a E_{1}+b E_{2}$ and $Y=Y^{\prime}+d E_{3}$ with $Y^{\prime} \in \mathfrak{z}^{\perp}$. Then, from (i) in the proof of Lemma 2.2, we get

$$
\begin{aligned}
\langle R(X, Y) Y, X\rangle= & \left|X \wedge Y^{\prime}\right|^{2} K\left(e_{1}, e_{2}\right) \\
& +\frac{d^{2}}{4}\left(a^{2} \frac{\beta^{2}}{\varepsilon^{2}} K\left(e_{1}, e_{3}\right)+b^{2} \frac{\alpha^{2}}{\varepsilon^{2}} K\left(e_{2}, e_{3}\right)\right) .
\end{aligned}
$$

Substituting for $K\left(e_{1}, e_{2}\right), K\left(e_{1}, e_{3}\right)$ and $K\left(e_{2}, e_{3}\right)$ and taking into account that the metric was multiplied by $4 \alpha^{2} \beta^{2} / \varepsilon^{2}$, we get

$$
\begin{aligned}
\langle R(X, Y) Y, X\rangle= & \frac{\Delta^{2}}{4 \varepsilon^{2}}\left(-\frac{3}{4} \varepsilon^{2}+\gamma^{2}-\alpha \beta\right) \\
+\frac{d^{2}}{16}[ & \frac{a^{2}}{\alpha^{2}}\left(\frac{1}{4} \varepsilon^{2}-\alpha(\alpha+\beta)\right) \\
& \left.+\frac{b^{2}}{\beta^{2}}\left(\frac{1}{4} \varepsilon^{2}-\beta(\alpha+\beta)\right)\right]
\end{aligned}
$$


where $\Delta^{2}$, defined by the expression $\left|X \wedge Y^{\prime}\right|^{2}=\left(\alpha^{2} \beta^{2} / \varepsilon^{4}\right) \Delta^{2}$ does not depend on $(\alpha, \beta, \gamma, \varepsilon)$.

If we write $[H, Y]-[T, X]=r E_{1}+s E_{2}+t E_{3}$, we have

$$
|[H, Y]-[T, X]|^{2}=r^{2} \frac{\beta^{2}}{\varepsilon^{2}}+s^{2} \frac{\alpha^{2}}{\varepsilon^{2}}+\frac{t^{2}}{4} \text {. }
$$

Therefore, if $R_{0}$ denotes the curvature tensor associated to the metric $\langle,\rangle_{0}$, we get

$$
\begin{aligned}
\langle R(X & +H, Y+T)(Y+T), X+H\rangle \\
& -\left\langle R_{0}(X+H, Y+T)(Y+T), X+H\right\rangle_{0} \\
= & \langle R(X, Y) Y, X\rangle-\left\langle R_{0}(X, Y) Y, X\right\rangle_{0} \\
& -|[H, Y]-[T, X]|^{2}+|[H, Y]-[T, X]|_{0}^{2} \\
= & \frac{\Delta^{2}}{4}\left(\frac{\gamma^{2}-\alpha \beta}{\varepsilon^{2}}-\frac{\gamma_{0}^{2}-\alpha_{0} \beta_{0}}{\varepsilon_{0}^{2}}\right) \\
& +\frac{d^{2}}{16}\left[\frac{a^{2}}{4}\left(\frac{\varepsilon^{2}}{\alpha^{2}}-\frac{\varepsilon_{0}^{2}}{\alpha_{0}^{2}}\right)+\frac{b^{2}}{4}\left(\frac{\varepsilon^{2}}{\beta^{2}}-\frac{\varepsilon_{0}^{2}}{\beta_{0}^{2}}\right)\right. \\
& \left.\quad-a^{2}\left(\frac{\beta}{\alpha}-\frac{\beta_{0}}{\alpha_{0}}\right)-b^{2}\left(\frac{\alpha}{\beta}-\frac{\alpha_{0}}{\beta_{0}}\right)\right] \\
& +r^{2}\left(\frac{\beta_{0}^{2}}{\varepsilon_{0}^{2}}-\frac{\beta^{2}}{\varepsilon^{2}}\right)+s^{2}\left(\frac{\alpha_{0}^{2}}{\varepsilon_{0}^{2}}-\frac{\alpha^{2}}{\varepsilon^{2}}\right) .
\end{aligned}
$$

Now, if we choose $\alpha_{0}=\beta_{0}, \varepsilon_{0}=2 \alpha_{0}$ and $\gamma_{0}^{2}=3 \alpha_{0}^{2}$, the right hand side of $(*)$ becomes

$$
\begin{aligned}
= & \frac{\Delta^{2}}{4}\left(\frac{\gamma^{2}-\alpha \beta}{\varepsilon^{2}}-\frac{1}{2}\right)+\frac{d^{2}}{16}\left(\frac{a^{2}}{\alpha^{2}}+\frac{b^{2}}{\beta^{2}}\right)\left(\frac{\varepsilon^{2}}{4}-\alpha \beta\right) \\
& +r^{2}\left(\frac{1}{4}-\frac{\beta^{2}}{\varepsilon^{2}}\right)+s^{2}\left(\frac{1}{4}-\frac{\alpha^{2}}{\varepsilon^{2}}\right) .
\end{aligned}
$$

Hence, if $(\alpha, \beta, \gamma, \varepsilon)$ satisfies the conditions $\varepsilon \leq 2 \alpha, \varepsilon \leq 2 \beta$ and $\gamma^{2} \leq \varepsilon^{2} / 2+\alpha \beta$, it follows that

$$
\begin{aligned}
& \langle R(X+H, Y+T)(Y+T), X+H\rangle \\
& \quad-\left\langle R_{0}(X+H, Y+T)(Y+T), X+H\right\rangle_{0} \leq 0 .
\end{aligned}
$$

If $R(\pi)=\langle R(X+H, Y+T)(Y+T), X+H\rangle$, where $\pi$ is the plane spanned by $\{X+H, Y+T\}$, we get $K \leq 0$ and the stronger condition $R(\pi) \leq R_{0}(\pi)$ for every plane $\pi \subset \mathfrak{g}$.

Conversely, if $R(\pi) \leq R_{0}(\pi)$ for all plane $\pi \subset \mathfrak{g}$, considering the planes spanned by $\{X, Y\}\left(X, Y \in z^{\perp}\right),\left\{e_{1}+T, e_{1}+H\right\}$ and 
$\left\{e_{2}+T, e_{2}+H\right\} \quad\left(T\right.$ and $H$ such that $\left.\lambda_{1}(T) \neq \lambda_{1}(H)\right)$ respectively, we get in each case $\gamma^{2} \leq \varepsilon^{2} / 2+\alpha \beta, \varepsilon \leq 2 \beta, \varepsilon \leq 2 \alpha$. Thus, we have the following:

Proposition 3.2. Let $G$ be the simply connected Lie group of $3 \times 3$ upper triangular real matrices of determinant one with the left invariant metric associated to $(\alpha, \beta, \gamma, \varepsilon)$. Then, $R(\pi) \leq R_{0}(\pi)$ for all plane $\pi \subset \mathfrak{g}$ if and only if $\gamma^{2} \leq \varepsilon^{2} / 2+\alpha \beta, \varepsilon \leq 2 \alpha$ and $\varepsilon \leq 2 \beta$. Moreover, $R(\pi)=R_{0}(\pi)$ for all plane $\pi \subset \mathfrak{g}$ if and only if $\gamma^{2}=\varepsilon^{2} / 2+\alpha \beta$, $\varepsilon=2 \alpha$ and $\varepsilon=2 \beta$ (that is, $G$ is symmetric).

In particular, $G$ is not symmetric if $R(\pi)<R_{0}(\pi)$ for some plane $\pi \subset \mathfrak{g}$.

\subsection{It follows from Theorem 2.7 that:}

(i) $G$ admits many different metrics of nonpositive curvature of rank one and only one metric, up to multiplication by a positive constant, of rank two. So the rank in a homogeneous space is not invariant under the change of homogeneous metrics of nonpositive curvature. This situation does not occur for Hadamard manifolds which are compact or have finite volume (see [4]).

(ii) $G$ with the left invariant metrics of rank one, gives us examples of homogeneous spaces of rank one having two-flats. In fact, $A=$ $\exp (\mathfrak{a})$ is a flat totally geodesic submanifold isometrically imbedded in $G$ of dimension two.

Acknowledgment. I would like to thank the referee for very detailed suggestions which helped to improve the exposition of this paper, shortening the proof of Theorem 2.1 and strengthening the statement of Theorem 2.7.

\section{REFERENCES}

[1] R. Azencott and E. Wilson, Homogeneous manifolds with negative curvature I, Trans. Amer. Math. Soc., 215 (1976), 323-362.

[2] __, Homogeneous manifolds with negative curvature II, Mem. Amer. Math. Soc., 178 (1976), 1-102.

[3] W. Ballmann, M. Brin and P. Eberlein, Structure of manifolds of nonpositive curvature I, Ann. of Math., 122 (1985), 171-203.

[4] W. Ballmann and P. Eberlein, Fundamental group of manifolds of nonpositive curvature, J. Differential Geom. 25 (1987), 1-22.

[5] M. J. Druetta, Homogeneous Riemannian manifolds and the visibility axiom, Geom. Dedicata, 17 (1985), 239-251.

[6] Visibility and rank one in homogeneous spaces of $K \leq 0$, Trans. Amer. Math. Soc., 304 (1987), 307-321. 
[7] M. J. Druetta, The rank in homogeneous spaces of nonpositive curvature, Proc. Amer. Math. Soc., 105 (1989), 972-978.

[8] _ - Fixed points of isometries at infinity in homogeneous spaces, Illinois $\mathrm{J}$. Math., 33 (2) (1989), 210-226.

[9] M. Salvai, Report to CONICET.

[10] J. Wolf, Homogeneity and bounded isometries in manifolds of negative curvature, Illinois J. Math., 8 (1964), 14-18.

Received April 11, 1989 and in revised form January 31, 1990. Supported in part by CONICOR.

UNIVERSIDAD NACIONAL DE CÓRDOBa

VALPARAISO Y ROGELIO MARTINEZ

CIUDAD UNIVERSITARIA

5000 Córdoba, ARgentina 



\section{PACIFIC JOURNAL OF MATHEMATICS EDITORS}

\author{
V. S. VARADARAJAN \\ (Managing Editor) \\ University of California \\ Los Angeles, CA 90024-1555-05 \\ Herbert Clemens \\ University of Utah \\ Salt Lake City, UT 84112 \\ THOMAS ENRIGHT \\ University of California, San Diego \\ La Jolla, CA 92093
}

R. FINN

Stanford University

Stanford, CA 94305

HeRmanN FlaschKa

University of Arizona

Tucson, AZ 85721

VAUGHAN F. R. JONES

University of California

Berkeley, CA 94720

STEVEN KERCKHOFF

Stanford University

Stanford, CA 94305
C. C. MOORE

University of California

Berkeley, CA 94720

Martin ScharlemanN

University of California

Santa Barbara, CA 93106

\section{HAROLD STARK}

University of California, San Diego La Jolla, CA 92093

\section{ASSOCIATE EDITORS}
R. ARENS
E. F. BECKENBACH
B. H. NeumanN
F. WolF
K. YoshidA
(1906-1982)

(1904-1989)
TIONS

UNIVERSITY OF ARIZONA

UNIVERSITY OF BRITISH COLUMBIA

CALIFORNIA INSTITUTE OF TECHNOLOGY

UNIVERSITY OF CALIFORNIA

MONTANA STATE UNIVERSITY

UNIVERSITY OF NEVADA, RENO

NEW MEXICO STATE UNIVERSITY

OREGON STATE UNIVERSITY
UNIVERSITY OF OREGON

UNIVERSITY OF SOUTHERN CALIFORNIA

STANFORD UNIVERSITY

UNIVERSITY OF HAWAII

UNIVERSITY OF TOKYO

UNIVERSITY OF UTAH

WASHINGTON STATE UNIVERSITY

UNIVERSITY OF WASHINGTON 


\section{Pacific Journal of Mathematics}

\section{Vol. 148, No. $1 \quad$ March, 1991}

David Marion Arnold and Charles Irvin Vinsonhaler, Duality and

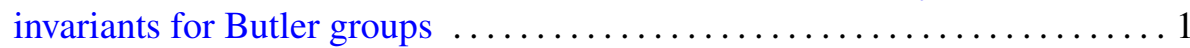

Philippe Delanoë, Obstruction to prescribed positive Ricci curvature . . . . . 11

María J. Druetta, Nonpositively curved homogeneous spaces of dimension

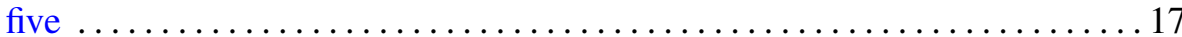

Robert Fitzgerald, Combinatorial techniques and abstract Witt rings III .... 39

Maria Girardi, Dentability, trees, and Dunford-Pettis operators on $L_{1} \ldots \ldots 59$

Krzysztof Jarosz, Ultraproducts and small bound perturbations $\ldots \ldots \ldots \ldots 81$

Russell David Lyons, The local structure of some measure-algebra homomorphisms .................................. 89

Fiona Anne Murnaghan, Asymptotic behaviour of supercuspidal characters of $p$-adic $\mathrm{GL}_{3}$ and $\mathrm{GL}_{4}$ : the generic unramified case $\ldots \ldots \ldots \ldots \ldots 107$

H. Rouhani, Quasi-rotation $C^{*}$-algebras $\ldots \ldots \ldots \ldots \ldots \ldots \ldots \ldots \ldots \ldots \ldots \ldots \ldots \ldots$

Ignacio Sols Lucía, Michał Szurek and Jaroslaw Wisniewski, Rank-2

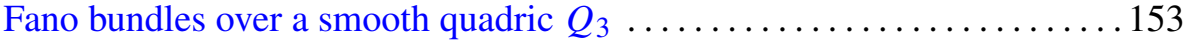

Martin Strake and Gerard Walschap, Ricci curvature and volume

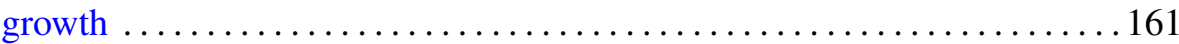

Anton Ströh and Johan Swart, A Riesz theory in von Neumann algebras . . 169

Ming Wang, The classification of flat compact complete space-forms with metric of signature $(2,2)$ 